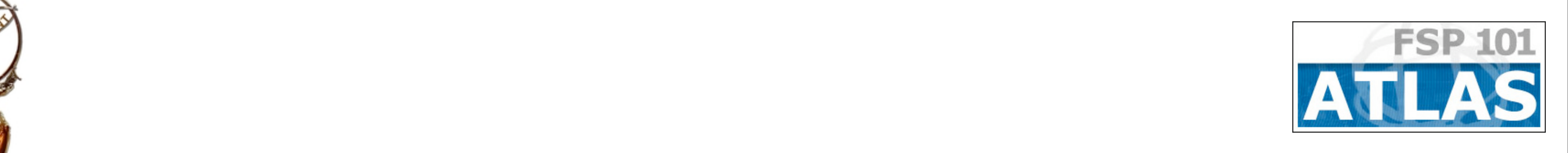

\title{
Tracking and Vertexing with the ATLAS Detector at the $\mathrm{LHC}$
}

Florian Hirsch on behalf of the ATLAS Collaboration PIXEL 2010, Grindelwald
GEFÖRDERT VOM 


\section{Outline}

- Overview of the tracking system

- Tracking and application

- algorithm description

- basic quantities

- application for b-tagging and physics

- Primary vertex finding and application

- algorithm description

- primary vertex quantities and interpretation

- Secondary vertices and b-tagging

- Further results with vertexing 


\section{ATLAS Inner Detector}

- Three-component tracking system immersed in a $2 \mathrm{~T}$ solenoidal magnetic field

- Silicon based Pixel Detector and Semiconductor Tracker (SCT)

- Drift tube based Transition Radiation Tracker (TRT)

- Excellent resolution of track kinematics

- Focus on silicon parts in this talk
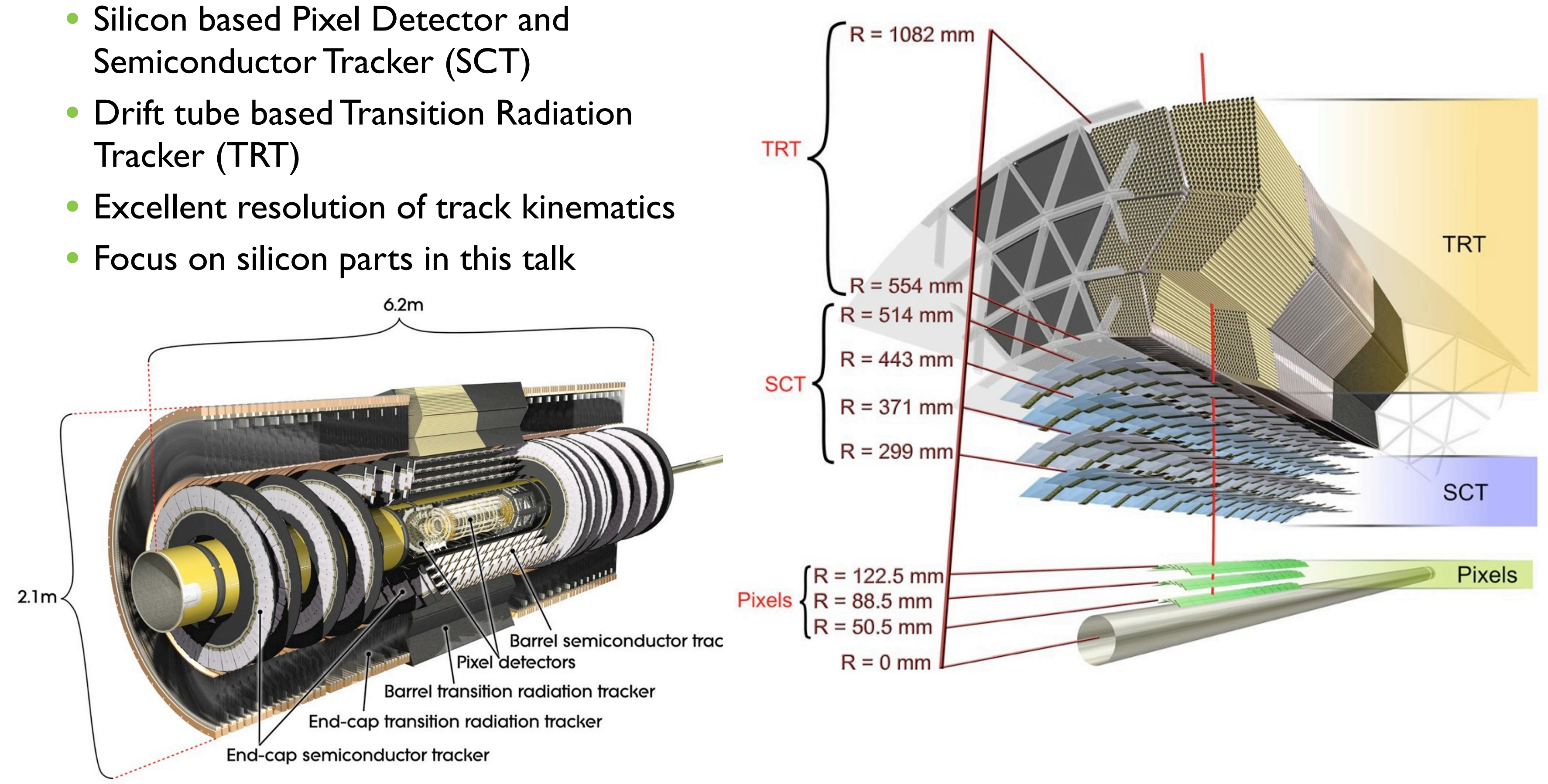


\section{Finding Tracks}

- Most commonly a pattern recognition algorithm seeded by hits in silicon detectors is used

- seeds are found in the silicon layers and a preliminary track direction is constructed; three space points in the silicon detectors are required to form a seed

- hits on these preliminary track paths are collected and a track candidate is fitted with an algorithm implementing a Kalman filter

- ambiguities between track candidates are solved with a pattern recognition algorithm assigning track scores based on hits-on-track and fit quality

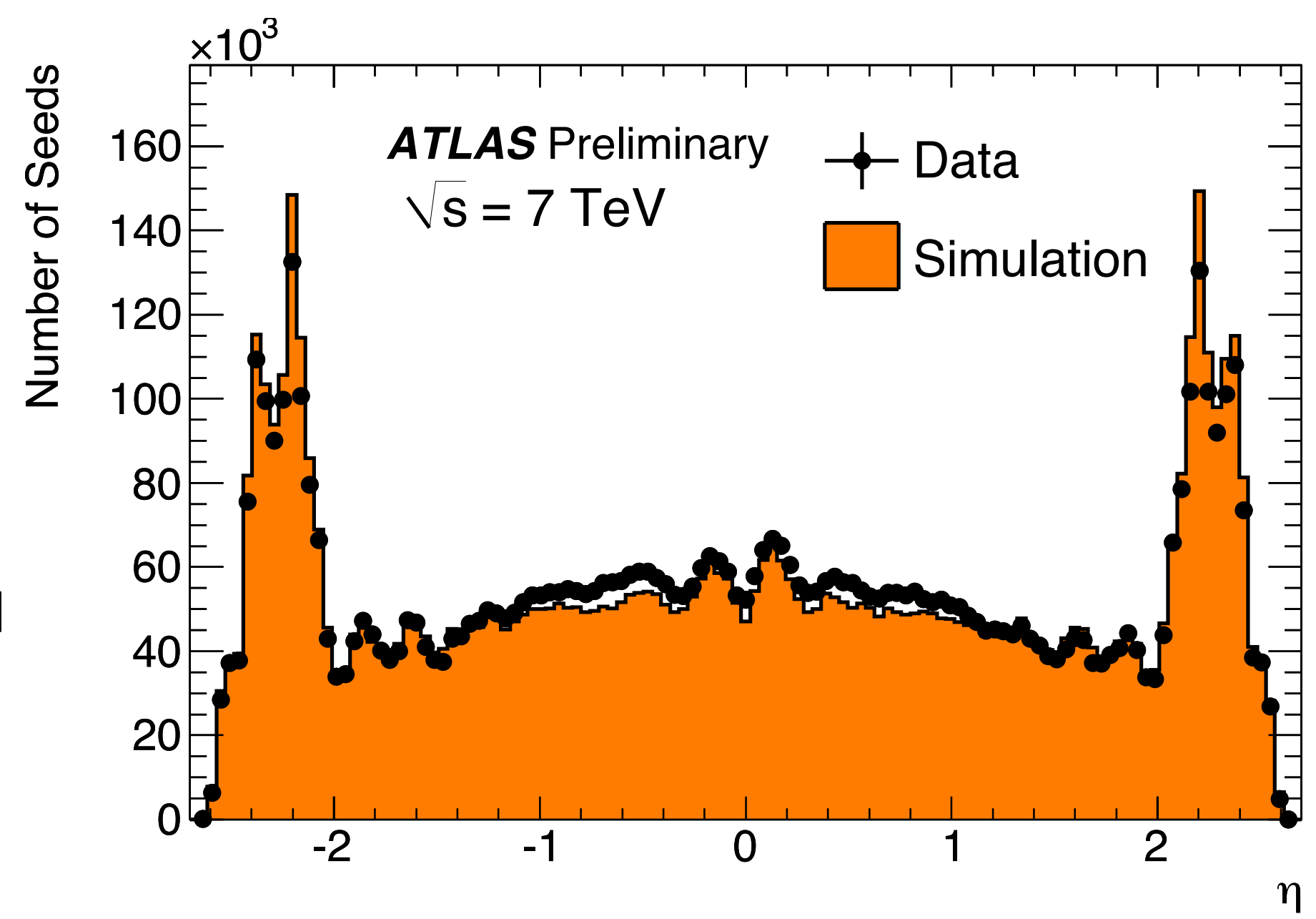




\section{Event Display with Tracks}

\section{QATLAS 道EXPERIMENT}

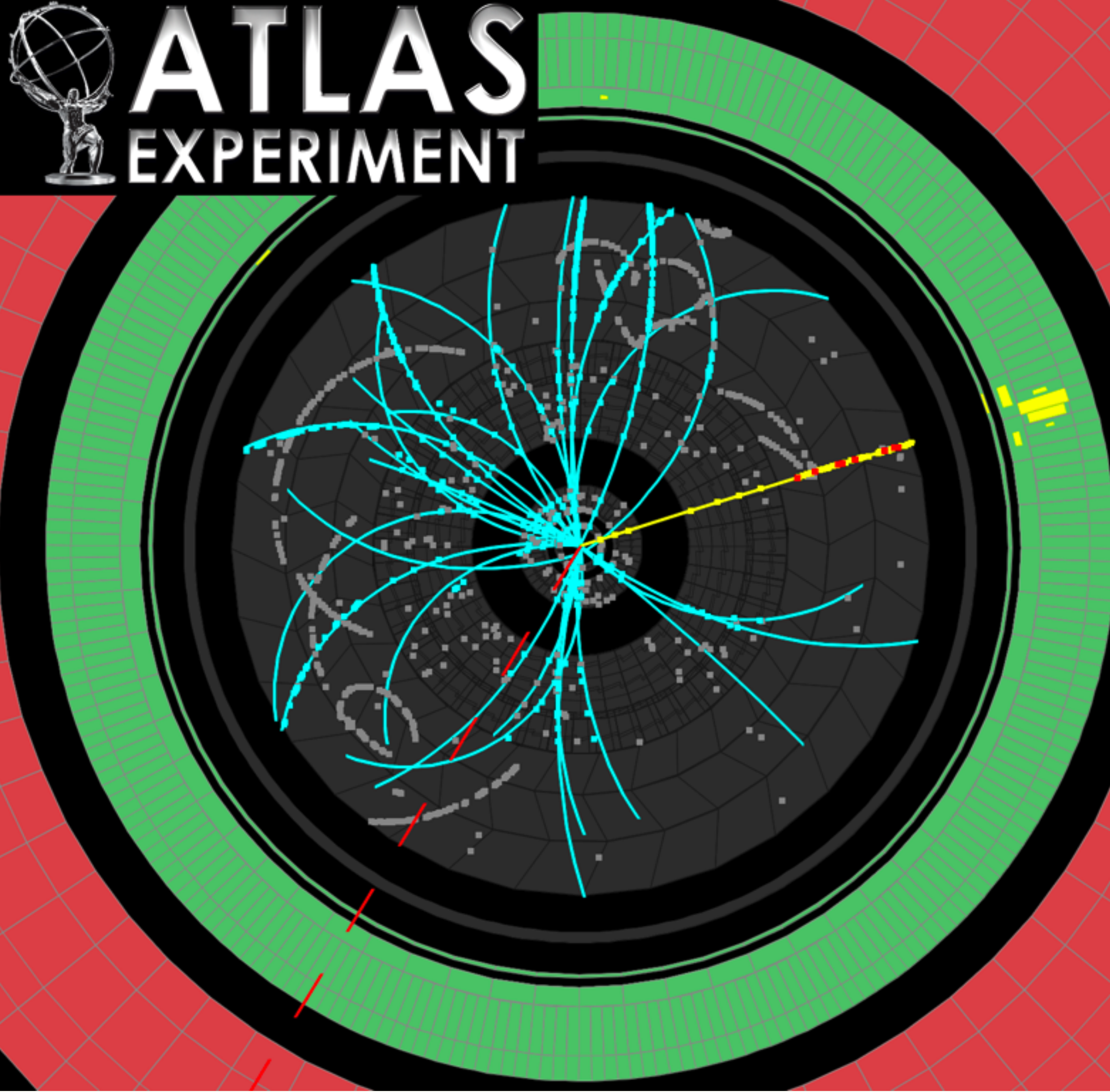

Run Number: 152409, Event Number: 5966801 Date: 2010-04-05 06:54:50 CEST

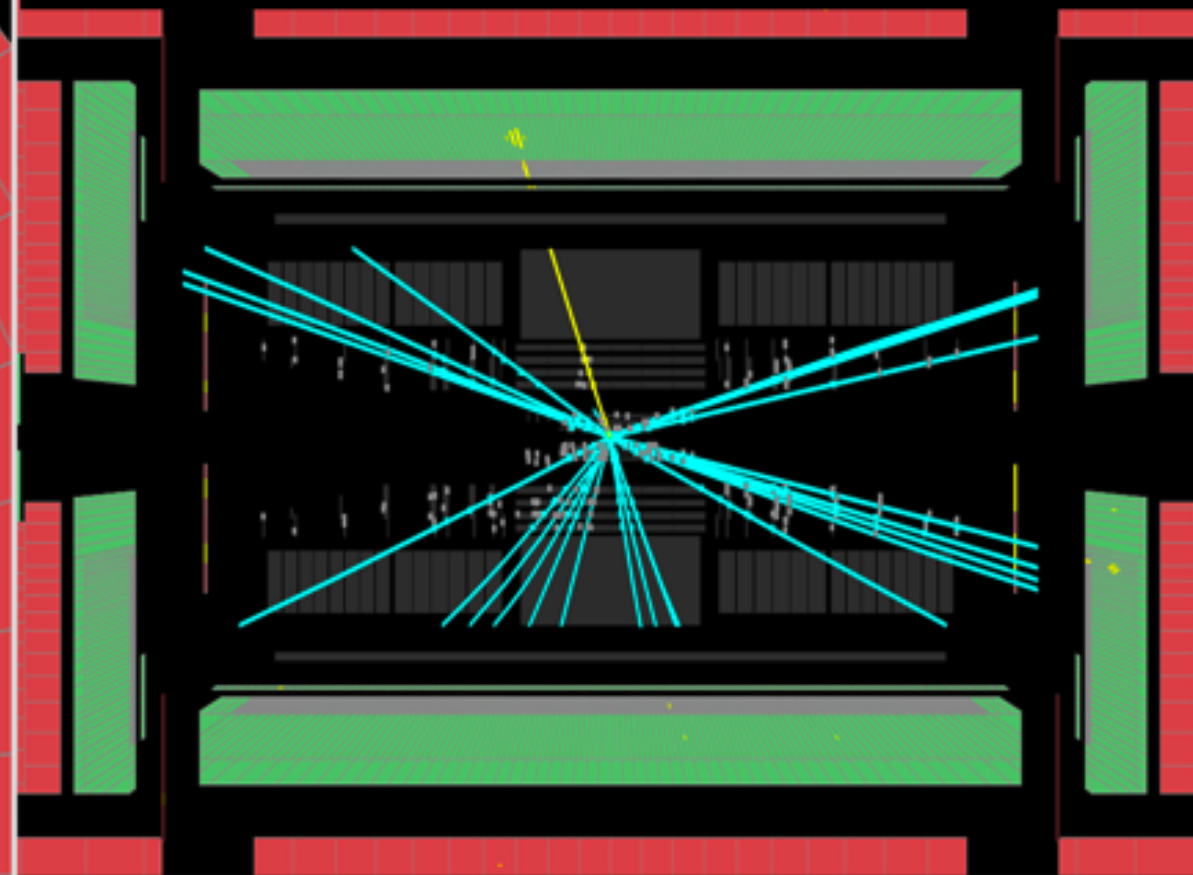

W $\rightarrow$ ev candidate in $7 \mathrm{TeV}$ collisions $\mathrm{p}_{\mathrm{T}}(\mathrm{e}+)=34 \mathrm{GeV}$ $\eta(e+)=-0.42$ $\mathrm{E}_{\mathrm{T}}^{\text {miss }}=26 \mathrm{GeV}$ $M_{\mathrm{T}}=57 \mathrm{GeV}$ 


\section{Hits on Track}

Tracks are built from Inner Detector hits. A different number of hits on a track is expected depending on the tracks' kinematics due to detector geometry and conditions.

To establish tracks and use them, it is therefore crucial to have a good understanding of the hit patterns.

Distributions of the number os hits-on-tracks show very good agreement for data and simulation.

Only tracks in jets are shown in these plots since they are of special importance for flavor tagging.
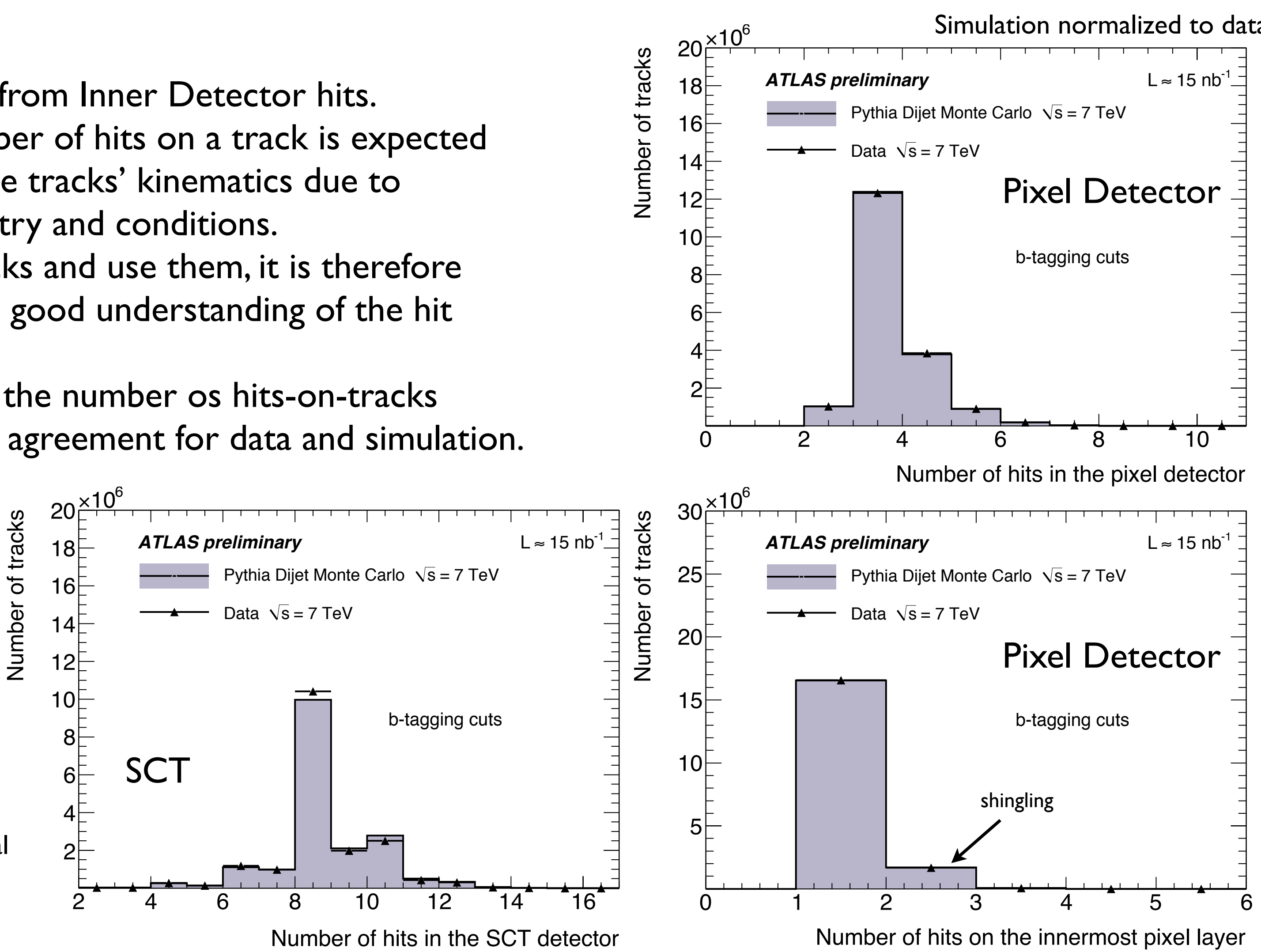


\section{Hits on Track}

A good description of data by simulation in different kinematic regions is important due to the detector layout.

Displayed are the average hit multiplicities in bins of eta and phi.

The agreement is good, the detector structure is well described by the simulation.

Only tracks in jets are shown in these plots since they are of special importance for flavor tagging.
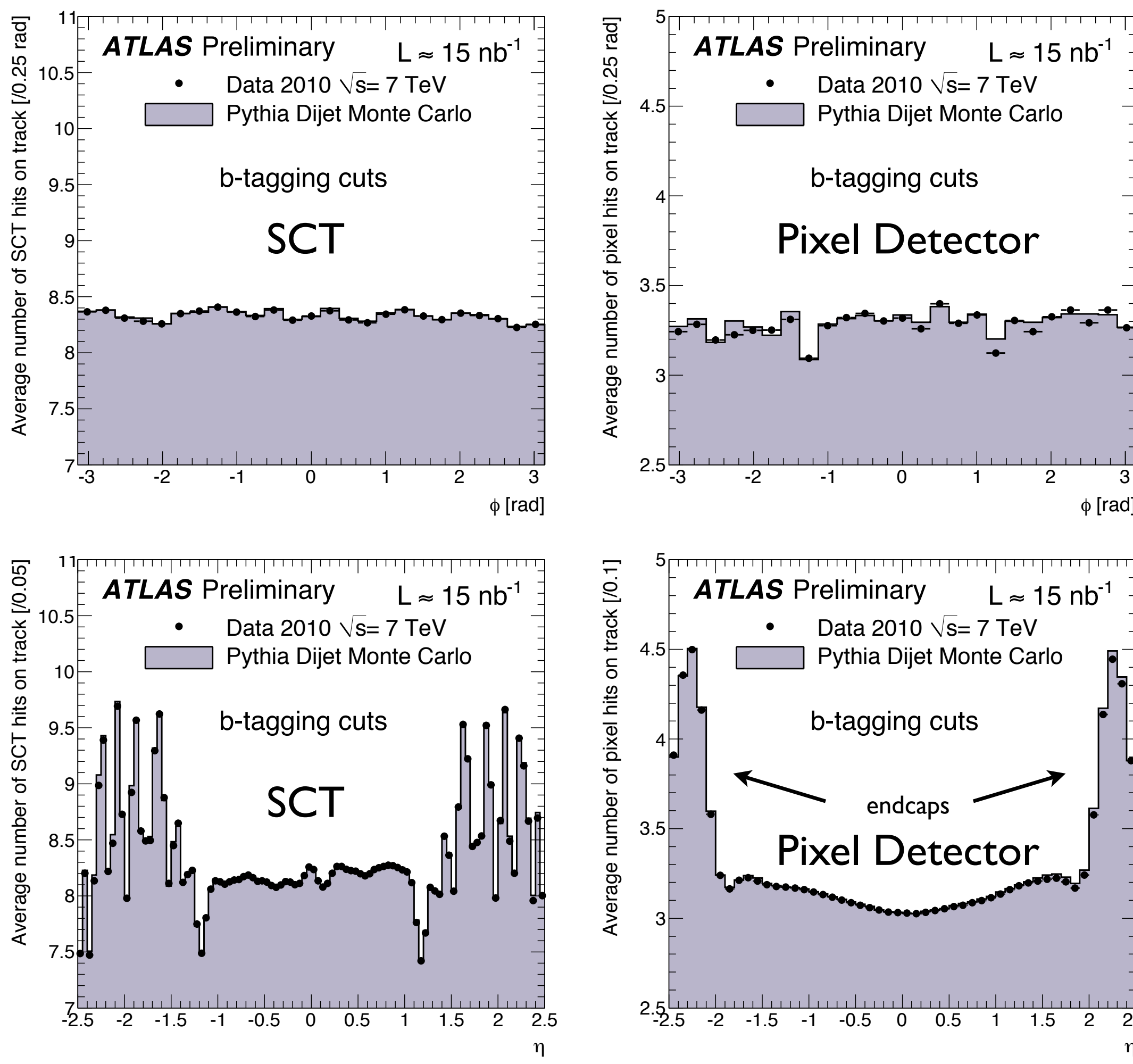


\section{Tracking for b-tagging}

Simulation normalized to data

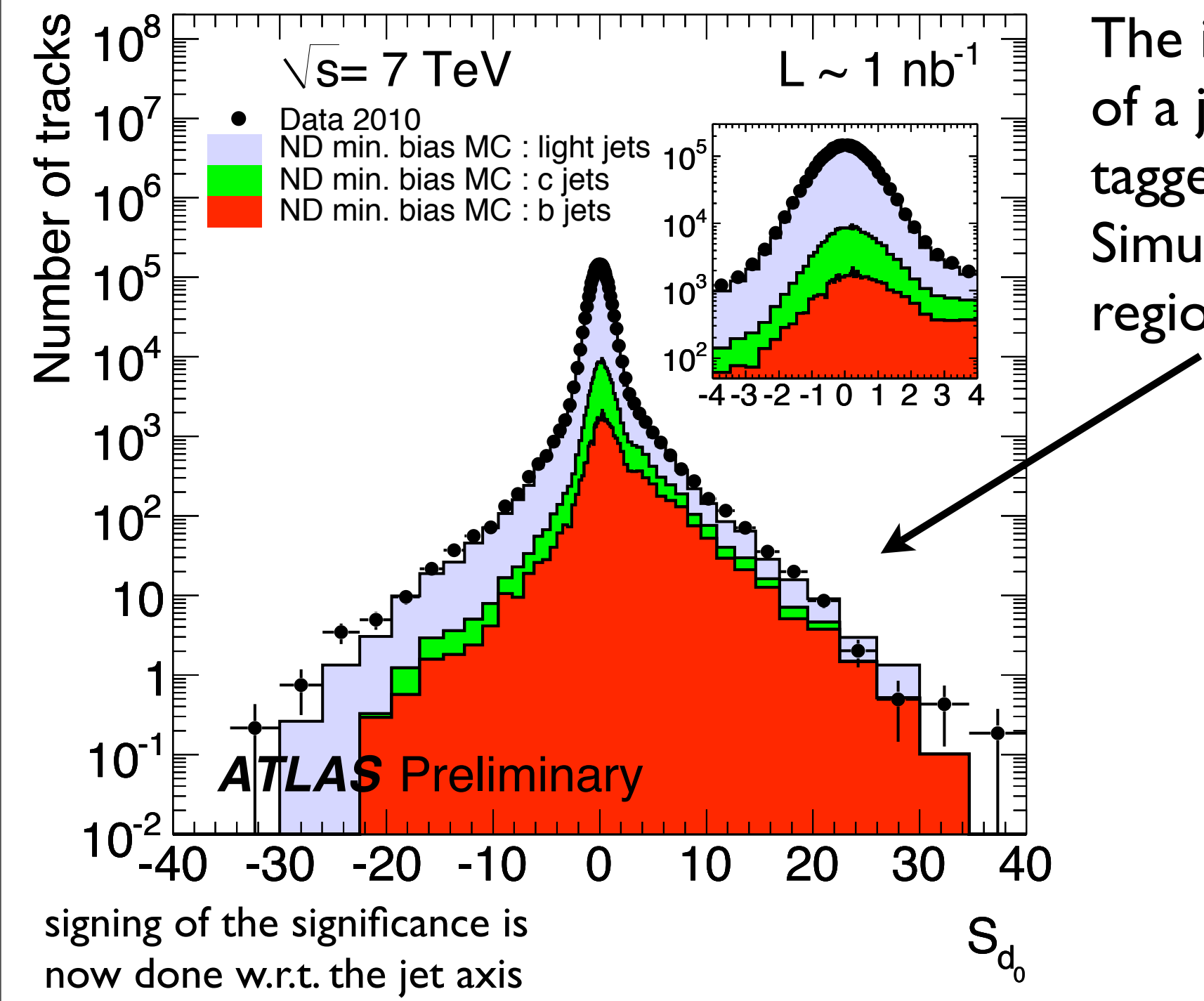

The probability of the jet being a light quark jet is derived from a calibration function which exploits the symmetry of the light jet distribution around 0 . It is clearly visible that a cut on this probability yields samples with increased b-content.

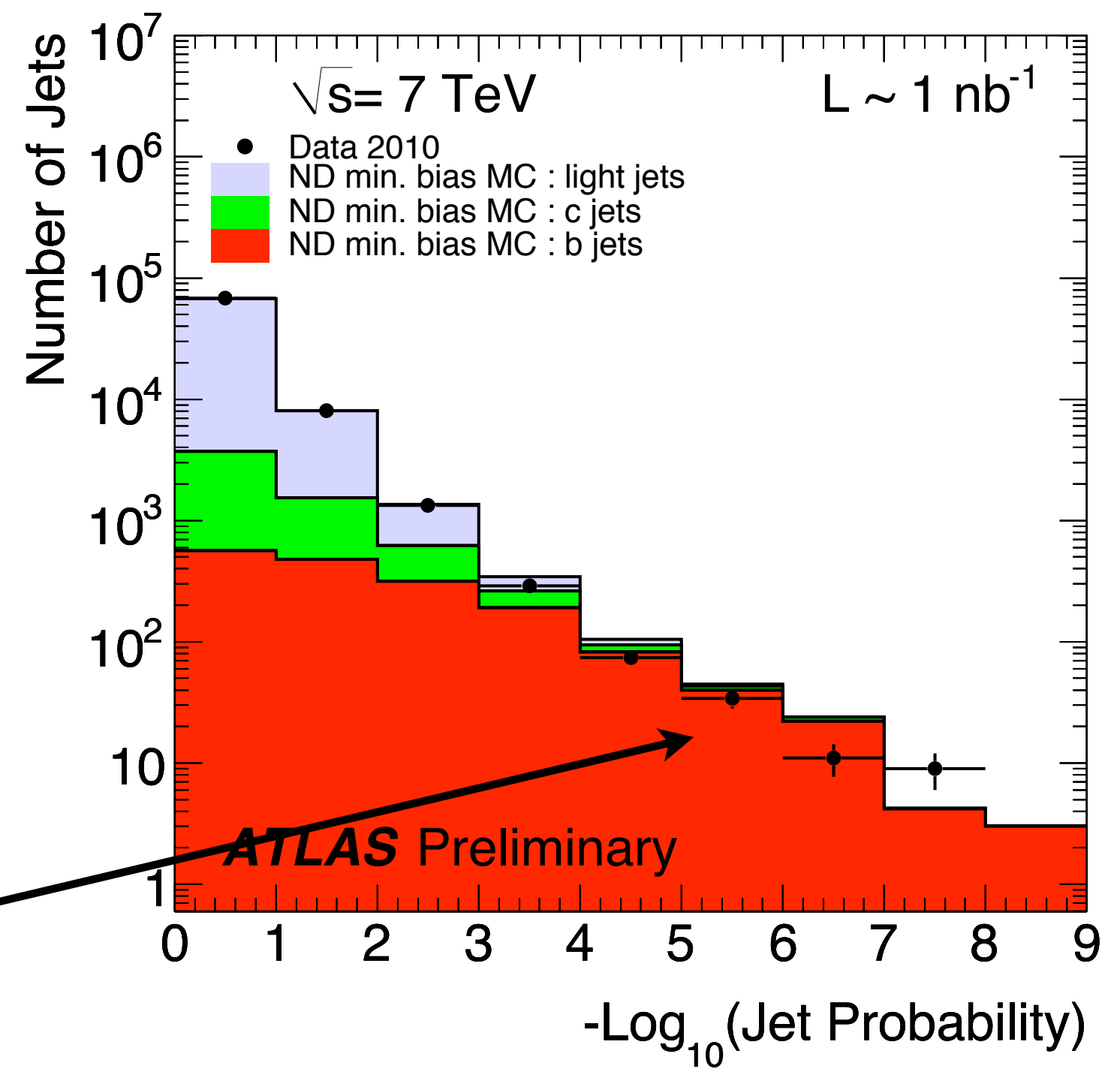




\section{Kinematic Properties of Tracks}

Another important track quantity is the transverse

momentum. The description of data by simulation is good and the track momenta can be used to form vertex masses.

The description of the track eta distribution by simulation is also shown and in good agreement with data.

ND $=$ non diffractive $\mathrm{SD}=$ single diffractive $\mathrm{DD}=$ double diffractive

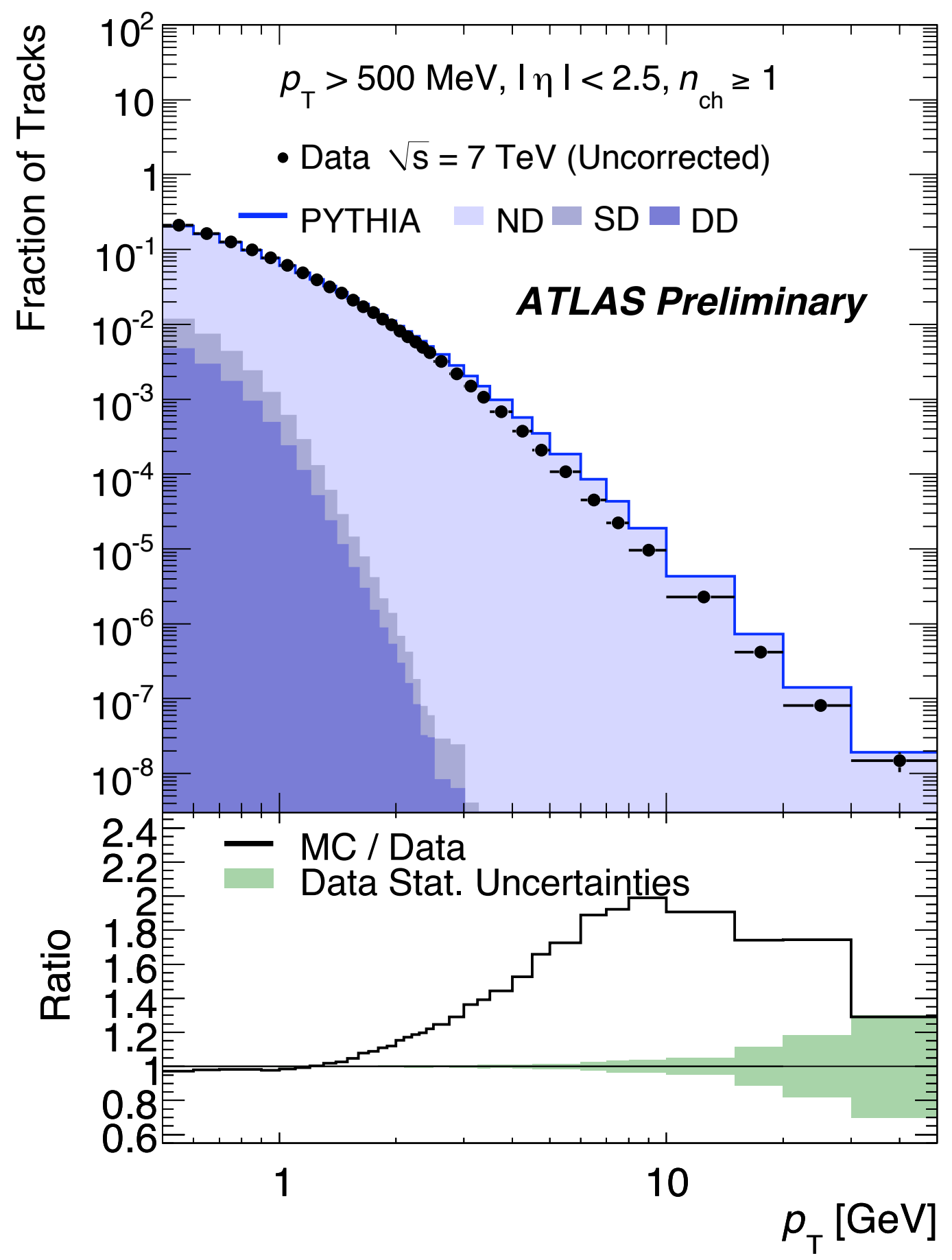

Normalized to unit area

Tracks from minimum bias events are shown.

They are no longer required to be in jets.

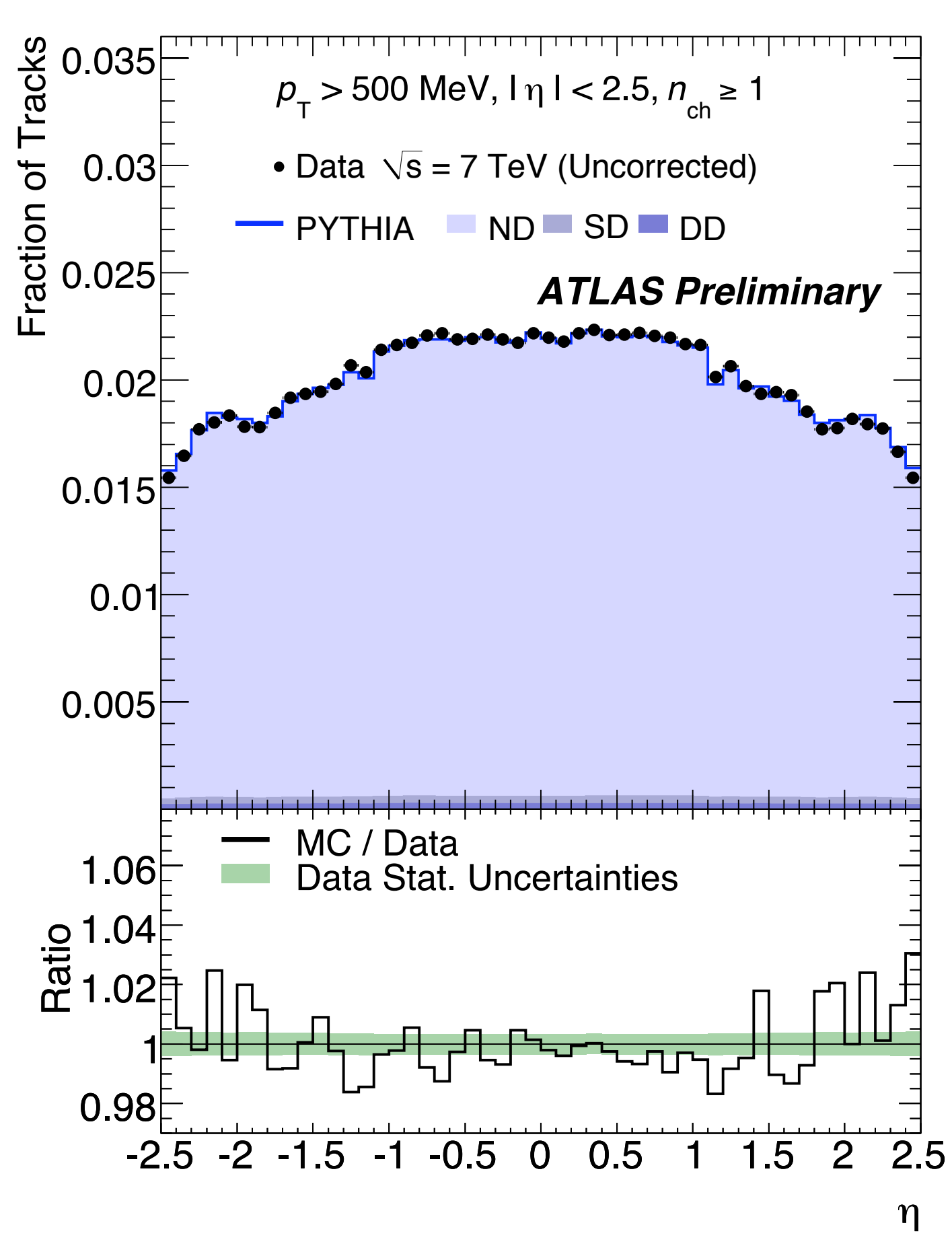




\section{Finding Primary Vertices}

- Primary vertices have to be reconstructed to

- measure the interaction point (vital for physics)

- measure luminous region

- measure pile-up

- A primary vertex is reconstructed in two steps

- a vertex seed is found by selecting tracks compatible with coming from the interaction region (beamspot constraint)

- an adaptive vertex fitter is applied to fit the vertex parameters from those tracks

- multiple vertices can be found by creating new seeds from tracks incompatible with the first primary vertex and iterating the algorithm 


\section{Beamspot Position}
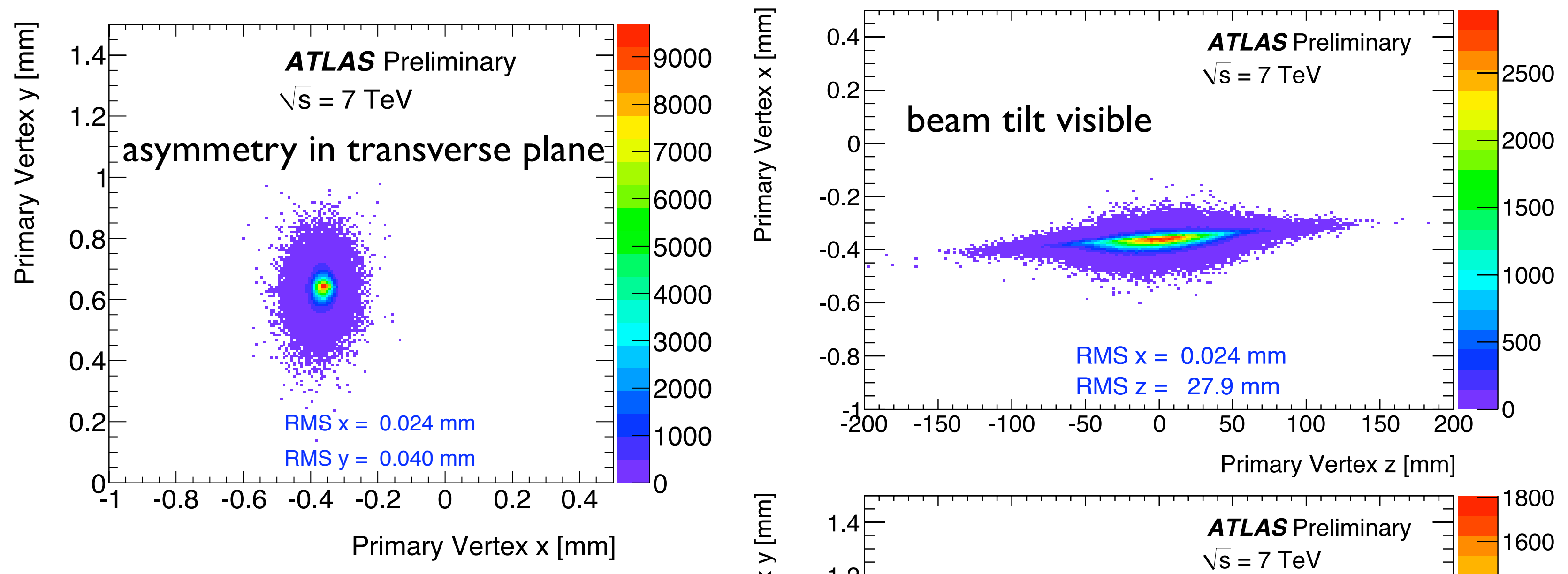

Reconstructed primary vertex position in 2D projections with beamspot constraint.

The distribution of primary vertices is used to measure the luminous region in ATLAS. In return this is used to constrain the primary vertex to the beam spot.

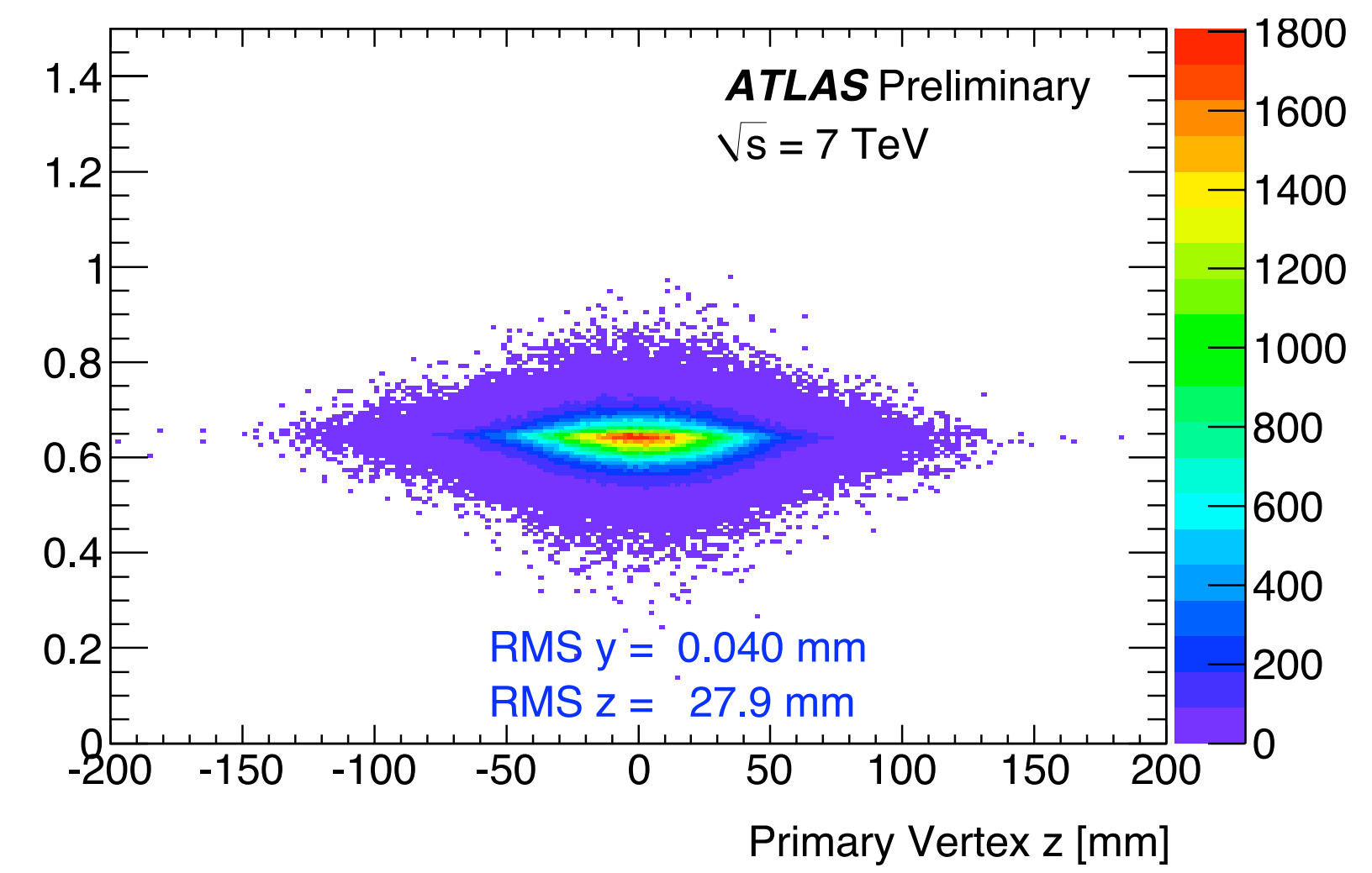




\section{Secondary Vertices for b-tagging}

jet cóne

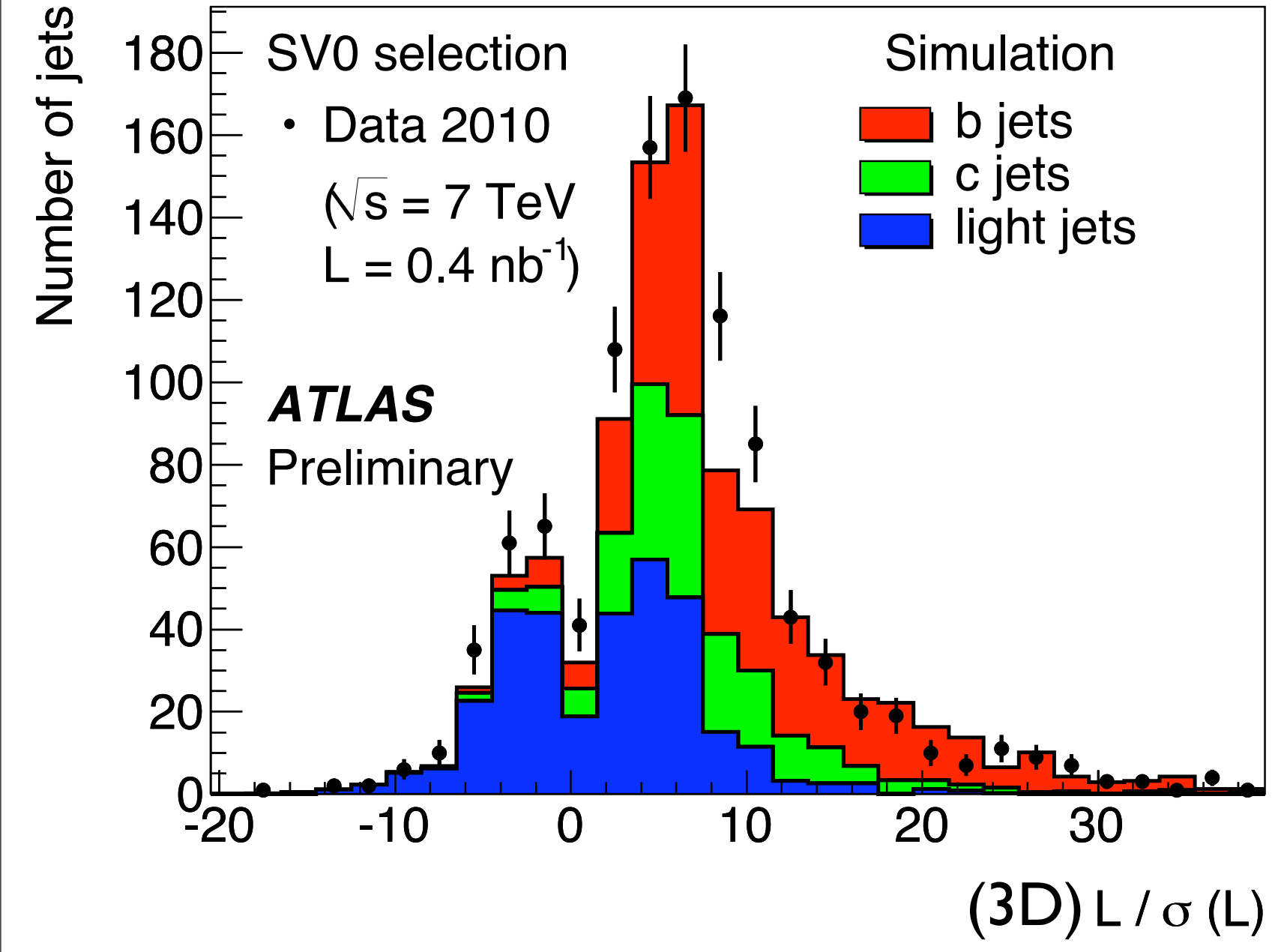

Secondary vertices can be used to identify particles with significant lifetimes.

This example shows how the SVO tagger enriches the sample with b-jets.
Simulation normalized

to number of jets in data
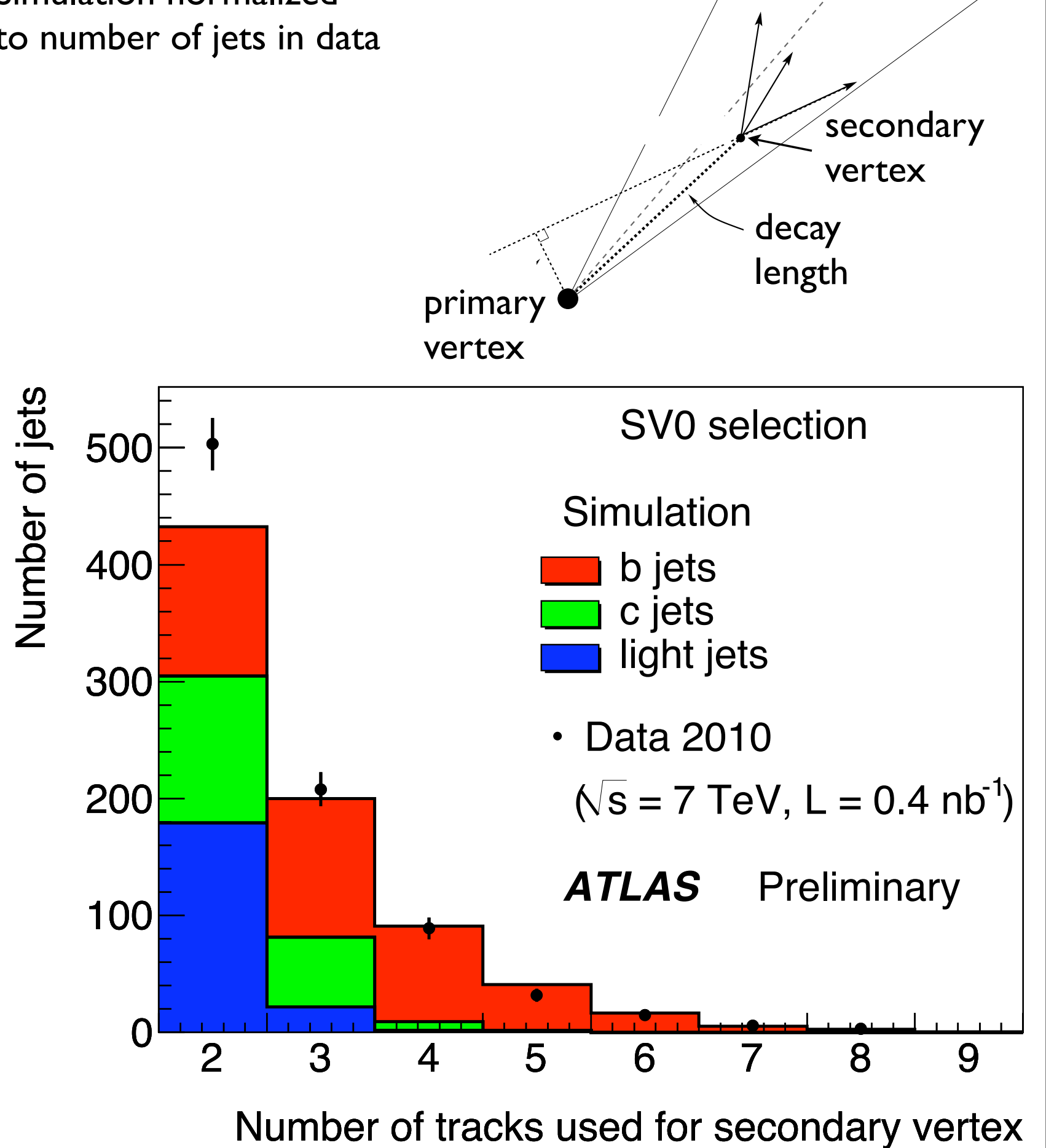


\section{More on Vertices}

Vertex reconstruction can also be used to map the material of the Pixel

Detector.

Here tracks compatible with the primary vertex or with $\gamma$-conversions or decays of light hadrons are rejected to find vertices from material interactions.

The beampipe and the different layers of the Pixel Detector are clearly visible as are services especially for the b-layer.
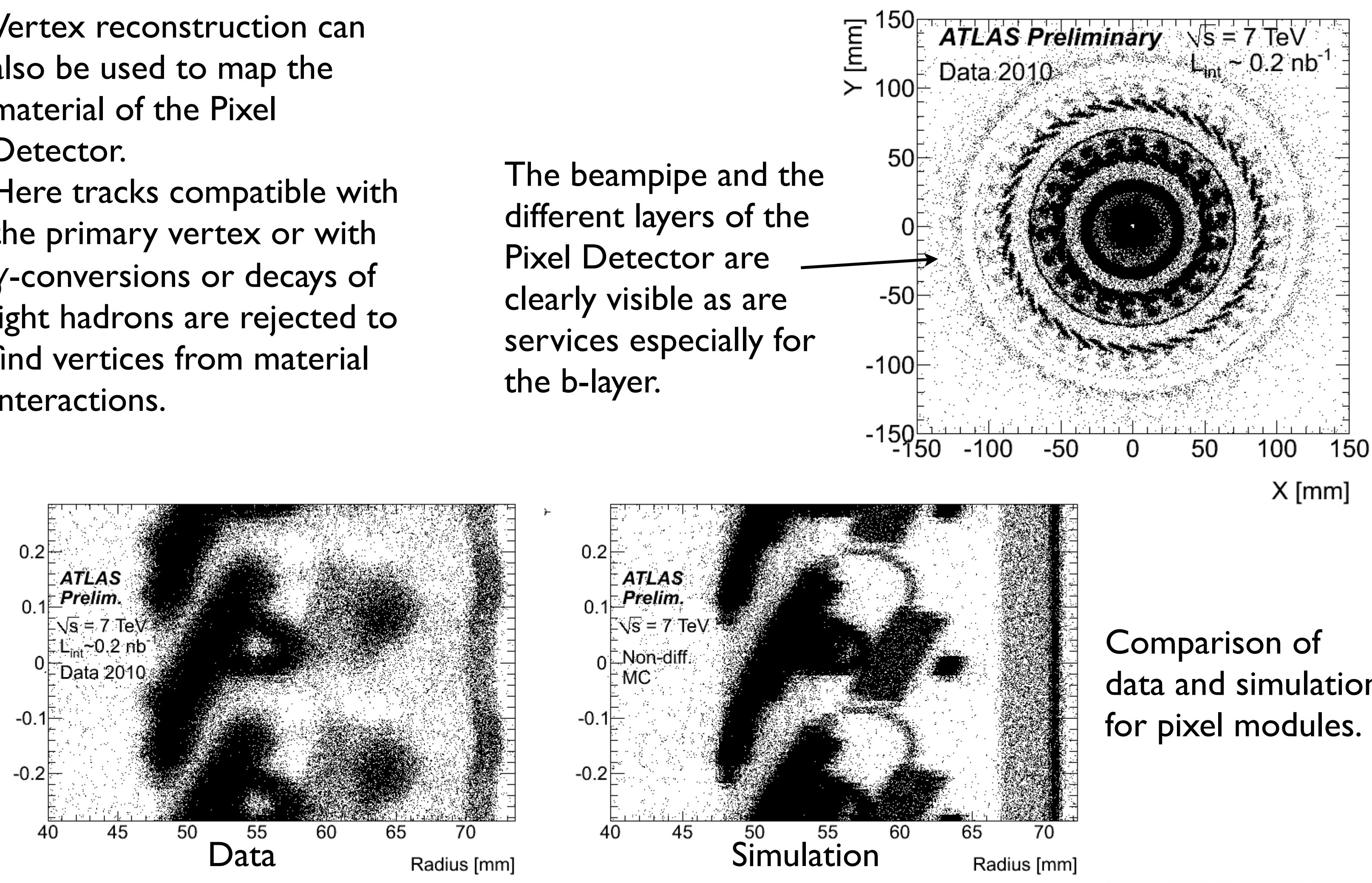

Comparison of data and simulation for pixel modules. 


\section{Summary}

- ATLAS features a three-component Inner Detector which is designed for tracking and vertexing

- we observe good agreement between data and Monte Carlo for basic quantities showing that we have a good understanding of the detector performance

- we also observe discrepancies in more sophisticated measurements which yield a lot of information about Monte Carlo tunings and the underlying physics

- Tracking and vertexing are vital for many applications

- they allow measurements of LHC conditions and a mapping of detector material

- also physics measurements like measuring the $\mathrm{J} / \Psi$ mass and the $\mathrm{J} / \Psi$ cross-section are possible and have been conducted

- flavor tagging algorithms depend strongly on precise track quantity measurements and an enhancement of heavy quark jets using these taggers has been shown 


\section{Backup}




\section{JetProb Tagger}

The JetProb tagger is a robust tagger which is being commissioned on early data.

It uses the shape of the negative side of the signed decay length distribution of tracks to derive a calibration function. The calibration function yields a per track likelihood that the track is a prompt track, the sum over those likelihoods gives a similar probability for the associated jet.

Jets with low JetProb probabilities are therefore likely to be b-jets.

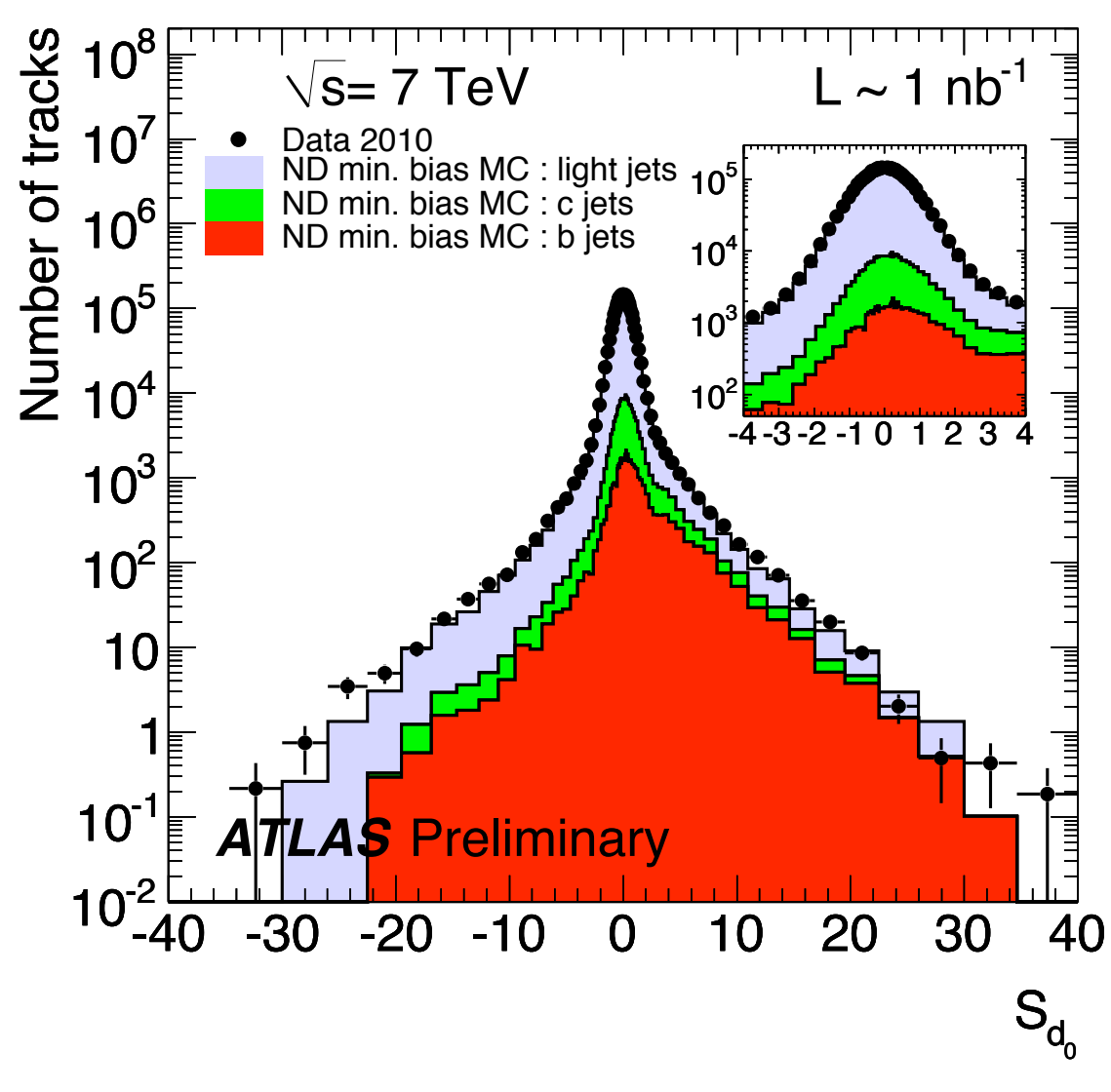$$
\text { 要 }
$$

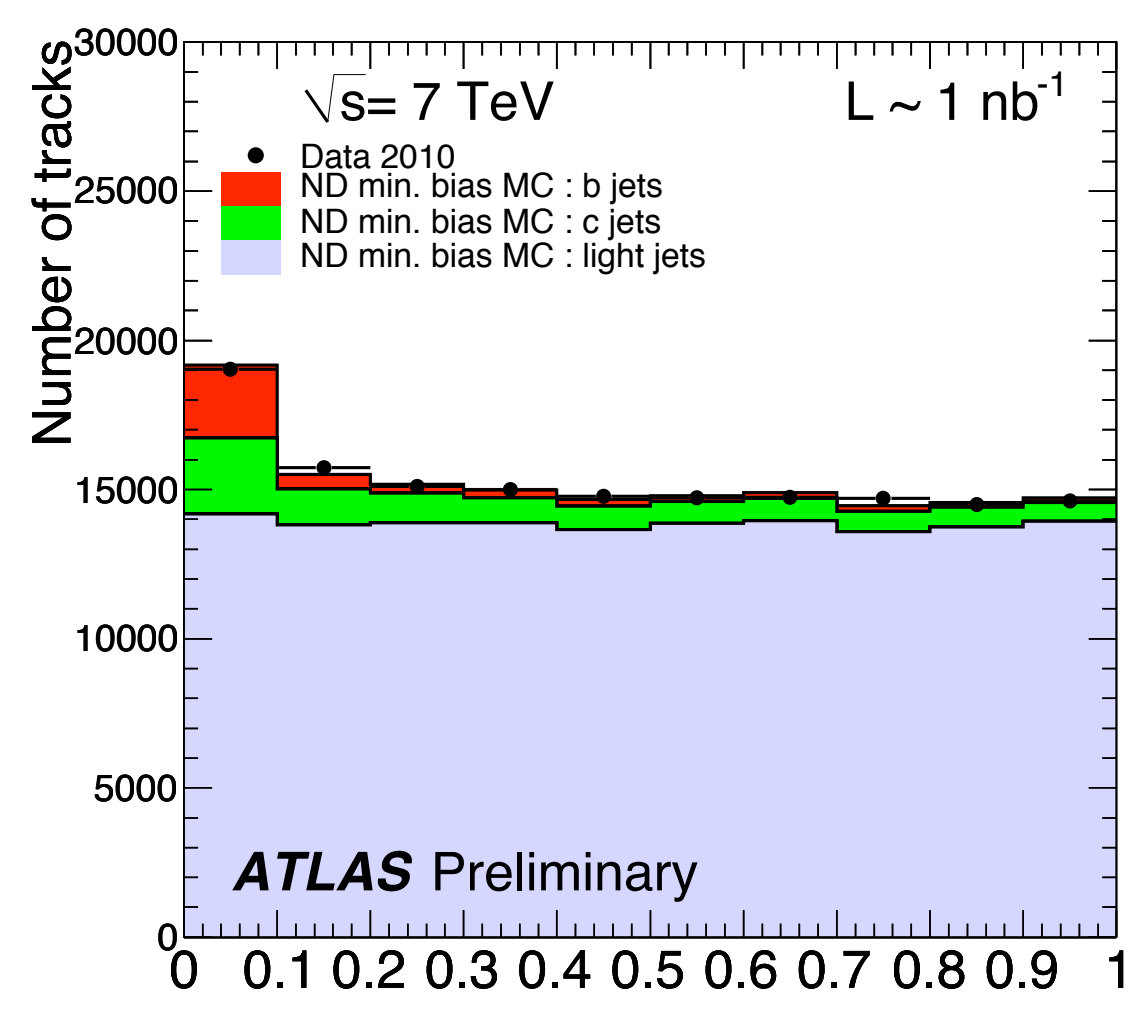

Track Probability 


\section{SVO Tagger}

The SV0 tagger is a robust tagger which is being commissioned on early data.

It uses a secondary vertex finder which rejects tracks compatible with the primary vertex and rejects vertices compatible with V0-decays, conversions and material interactions. The tagging weight is the signed decay length significance of the reconstructed secondary vertex (in the jet).

Vertices with a high signed decay length significance are likely b-decays.
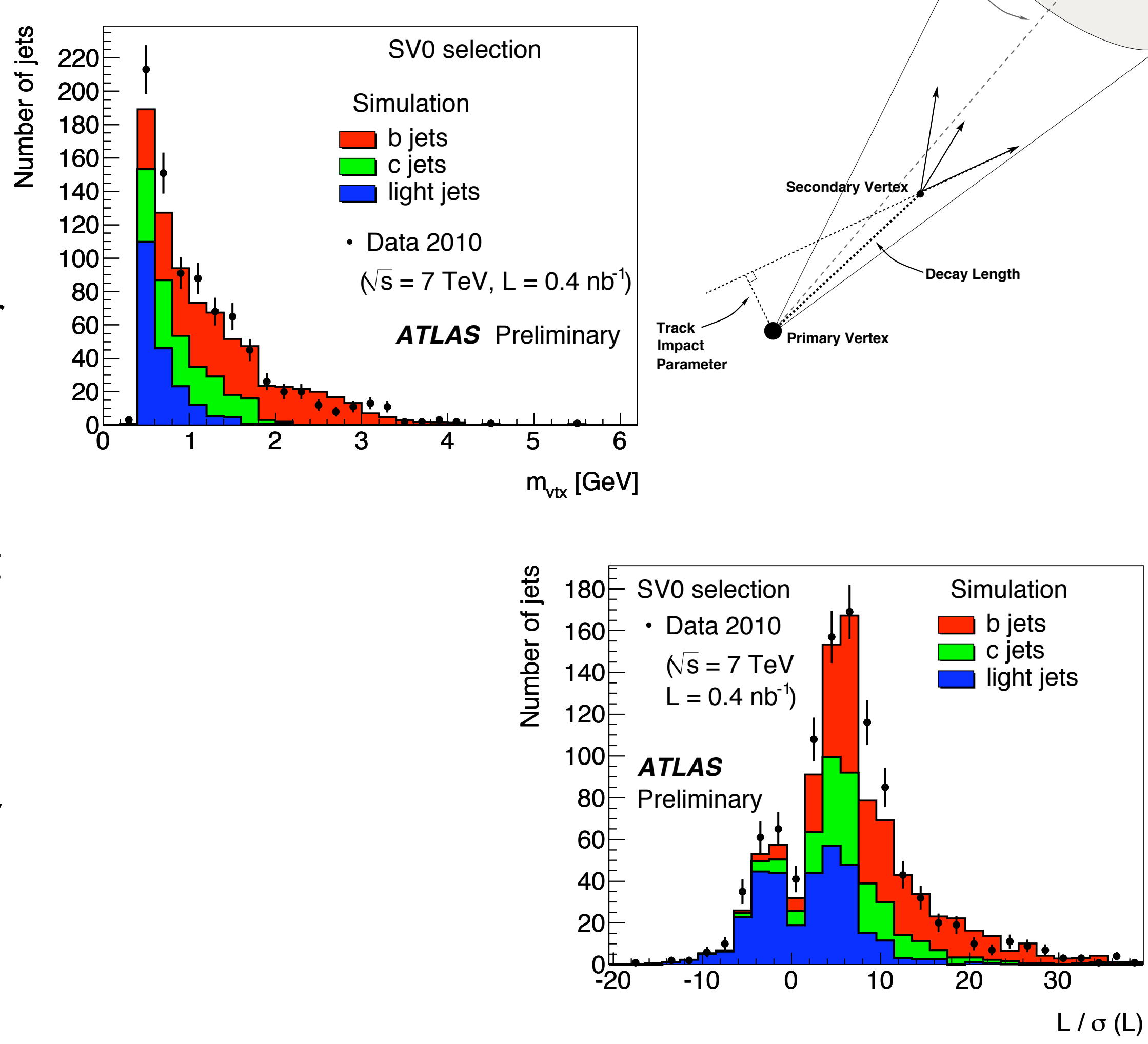